Mitteilungen der Deutschen Gesellschaft für Phlebologie

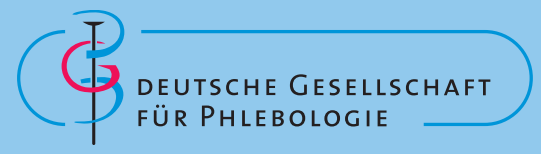

\title{
Empfehlungen zur Thromboseprophylaxe bei SARS-CoV-2 (COVID-19)
}

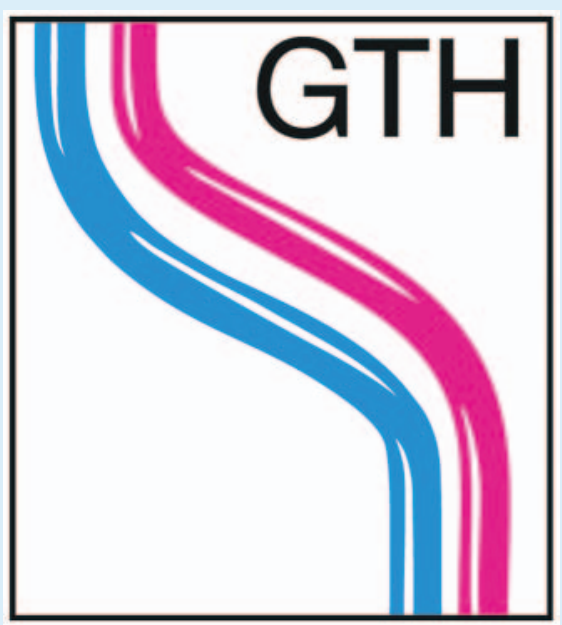

Erste Fallserien und Kohortenstudien zeigen, dass bei Patienten mit COVID-19 eine übermäßige Gerinnungsaktivierung bezüglich Krankenhaussterblichkeit und Notwendigkeit einer intensivmedizinischen Behandlung von prognostischer Relevanz ist [1-3]. Diese Daten werden ergänzt durch anekdotische Berichte über ein gehäuftes Auftreten von venösen Thromboembolien (VTE) bei ambulanten und stationären COVID-19-Patienten. Eine aktuelle retrospektive Analyse von 449 Patienten mit schwerer SARS-CoV-2-Infektion legt außerdem nahe, dass in den Subgruppen von Patienten mit septischer Koagulopathie (definiert durch die Parameter Thrombozytenzahl, Quick/INR und SOFA-Score) oder mit deutlich erhöhten D-Dimeren (>6-fache des oberen Referenzwertes) eine prophylaktische Antikoagulation $\geq 7$ Tage, vorwiegend mit niedermolekularem Heparin $(\mathrm{NMH})$, die 28-Tages-Mortalität reduziert [4].

Basierend auf der bisher verfügbaren Literatur und in Anlehnung an die ISTH [5] hat der GTH-Vorstand die folgenden Empfehlungen zur VTE-Prophylaxe bei Patienten mit SARS-CoV-2-Infektion (COVID-19) formuliert, die zwingend in regelmäßigen Abstän- den an den aktuellen Wissensstand anzupassen sind:

- Bei allen Patienten mit gesicherter SARS-CoV-2-Infektion sollte die Indikation zur medikamentösen VTE-Prophylaxe mit NMH unabhängig von der Notwendigkeit einer Hospitalisierung fortlaufend geprüft und großzügig gestellt werden.

- Ist eine Indikation zur medikamentösen VTE-Prophylaxe gegeben, sollte diese mit NMH in einer für den Hochrisikobereich zugelassenen Dosierungerfolgen. Liegen Kontraindikationen für eine Antikoagulation vor, sollten physikalische Maßnahmen (z. B. Kompressionsstrümpfe) zur Anwendung kommen.

- Bei Patienten mit gesicherter SARSCoV-2-Infektion und akuten Krankheitssymptomen ist eine Bestimmung der D-Dimere sinnvoll. Bei signifikant erhöhten D-Dimeren ( $\geq 1,5-2,0 \mathrm{mg} / \mathrm{l}$ ) ist eine medikamentöse Thromboseprophylaxe indiziert. Zudem sollte dann unabhängig von der Krankheitssymptomatik eine stationäre Aufnahme zur Überwachung erwogen werden.

- Alle hospitalisierten Patienten mit SARSCoV-2-Infektion sollten in Abwesenheit von Kontraindikationen eine medikamentöse VTE-Prophylaxe erhalten.

- Bei Vorliegen zusätzlicher Risikofaktoren (z. B. BMI > $30 \mathrm{~kg} / \mathrm{m}^{2}$, stattgehabte VTE, aktive Krebserkrankung), bei intensivmedizinisch behandelten Patienten und/ oder bei einem raschen Anstieg der D-Dimere sollte unter Berücksichtigung von Nierenfunktion und Blutungsrisiko eine intensivierte Thromboseprophylaxe erwogen werden (z. B. NMH in halbtherapeutischer Dosierung 1 x täglich oder $\mathrm{NMH}$ in prophylaktischer Dosierung 2 xtäglich).

- In Abwesenheit einer gesicherten Thromboembolie oder ECMO-Behandlung kann eine therapeutisch dosierte
Antikoagulation aktuell nicht routinemäßig empfohlen werden. Bei klinischem Verdacht auf eine VTE (z. B. akute Zunahme der Dyspnoe, unerklärter Abfall von Sauerstoffsättigung/-partialdruck, zunehmende Beinödeme, massiver Anstieg der D-Dimere) sollte jedoch die Indikation zur bildgebenden Diagnostik (CTPA, Sonographie) großzügig gestellt werden.

- Alle hospitalisierten Patienten mit SARSCoV-2-Infektion sollten fortlaufend hämostaseologisch überwacht werden. Sinnvolle Laborparameter sind: D-Dimere, Prothrombinzeit (Quick/INR), Thrombozytenzahl, Fibrinogen und Antithrombin.

- Bei Patienten mit SARS-CoV-2-Infektion stellen eine Thrombozytopenie und eine verlängerte APTT oder Prothrombinzeit ohne Blutungssymptome per se keine Kontraindikationen zur Durchführung einer medikamentösen VTE-Prophylaxe dar.

- Bei einer ECMO-Behandlung sollte unter Verwendung von unfraktioniertem Heparin (UFH) eine 1,5- bis 1,8-fache APTT-Verlängerung angestrebt werden.

- Bei fortbestehender Immobilität, hoher entzündlicher Aktivität und/oder zusätzlichen Risikofaktoren (siehe oben) ist nach Entlassung aus der stationären Behandlung eine prolongierte ambulante NMH-Prophylaxe sinnvoll und dann im Arztbrief entsprechend zu kommunizieren.

\section{Mit freundlicher Empfehlung}

Vorstandsvorsitzender der GTH

Prof. Dr. med. Johannes Oldenburg

Chairman and Director

University Clinic Bonn, AöR Institute of Experimental Haematology and Transfusion Medicine 
Stellv. Vorsitzender der GTH

Priv.- Doz. Dr. med. Robert Klamroth

Chefarzt der Klinik für Innere Medizin,

Angiologie und Hämostaseologie

Zentrum für Gefäßmedizin, Vivantes-Klini-

kum im Friedrichshain

\section{Schriftü̈hrer der GTH}

Prof. Dr. med. Florian Langer

Leiter des Bereichs Hämostaseologie (Gerinnungsambulanz und Hämophiliezentrum)

Universitätsklinikum Hamburg-Eppendorf, II. Medizinische Klinik und Poliklinik (Onkologie, Hämatologie und KMT mit der Sektion Pneumologie)

\section{Literatur}

[1] Guan WJ, Ni ZY, Hu Y et al. Clinical Characteristics of Coronavirus Disease 2019 in China. N Engl ] Med 2020 [Epub ahead of print]

[2] Huang C, Wang Y, Li X et al. Clinical features of patients infected with 2019 novel coronavirus in Wuhan, China. Lancet 2020; 395: 497-506

[3] Tang N, Li D, Wang X et al. Abnormal coagulation parameters areassociated with poor prognosis in patients with novel coronavirus pneumonia. ] Thromb Haemost 2020 [Epub ahead of print]

[4] Klok FA, Kruip MJHA, van der Meer NJM et al. Incidence of thrombotic complications in critically ill ICU patients with COVID-19. Thromb Res 2020 [Epub ahead of print]

[5] Cui S, Chen S, Li X et al. Prevalence of venous thromboembolism in patients with severe novel coronavirus pneumonia. J Thromb Haemost 2020 [Epub ahead of print]
[6] Tang N, Bai H, Chen X et al. Anticoagulant treatment is associated with decreased mortality in severe coronavirus disease 2019 patients with coagulopathy. J Thromb Haemost 2020 [Epub ahead of print]

[7] Thachil J, Tang N, Gando S et al. ISTH interim guidance on recognition and management of coagulopathy in COVID-19. J Thromb Haemost 2020 [Epub ahead of print]

[8] Casini A, Alberio L, Angelillo-Scherrer A et al. Thromboprophylaxis and laboratory monitoring for in-hospital patients with COVID-19 - a Swiss consensus statement by the Working Party Hemostasis. Swiss Med Wkly 2020; 150 : w20247

[9] Marietta M, Ageno W, Artoni A et al. COVID-19 and haemostasis: a position paper from Italian Society on Thrombosis and Haemostasis (SISET). Blood Transfus 2020 [Epub ahead of print] 